Hausdorff dimension of three-period orbits in Birkhoff billiards

This article has been downloaded from IOPscience. Please scroll down to see the full text article.

2012 Nonlinearity 251947

(http://iopscience.iop.org/0951-7715/25/7/1947)

View the table of contents for this issue, or go to the journal homepage for more

Download details:

IP Address: 130.126.108.82

The article was downloaded on 16/02/2013 at 18:09

Please note that terms and conditions apply. 


\title{
Hausdorff dimension of three-period orbits in Birkhoff billiards
}

\author{
Sergei Merenkov and Vadim Zharnitsky \\ Department of Mathematics, University of Illinois, 1409 W Green Street, Urbana, IL 61801, USA \\ E-mail: merenkov@illinois.edu and vz@math.uiuc.edu
}

Received 12 December 2011, in final form 21 May 2012

Published 13 June 2012

Online at stacks.iop.org/Non/25/1947

Recommended by D Dolgopyat

\begin{abstract}
We prove that the Hausdorff dimension of the set of three-period orbits in classical billiards is at most one. Moreover, if the set of three-period orbits has Hausdorff dimension one, then it has a tangent line at almost every point.
\end{abstract}

PACS numbers: 45.20.Jj, 47.10.Df

Mathematics Subject Classification: 37 j99

\section{Introduction}

The goal of this note is to provide a sharp estimate on the dimension of the set $P^{3}$ of three-period orbits in classical billiards. The question about Lebesgue measure of the set of periodic orbits was raised by Ivrii [4], in connection with spectral geometry problems. The conjecture that periodic orbits have zero measure is still open, but several special cases have been resolved. The first result was due to Rychlik [6] who proved that the set of three-period orbits has zero Lebesgue measure. More recently a proof for the four-period case has been announced in [2].

Consider a not necessarily convex billiard boundary and define the billiard map $T$ in the standard way. The boundary of length $l$ is parametrized with a natural parameter $t$. For an outgoing trajectory, let $\theta$ be the angle with the tangent. Then the billiard map $T\left(t_{1}, \theta_{1}\right)=\left(t_{2}, \theta_{2}\right)$ is defined for any segment that is transversal to the boundary at both ends. We disregard those that are not, since they have zero measure. The billiard map is defined on an open annulus $U=S^{1} \times I$, where $S^{1}$ is the boundary of length $l$ and $I=(0, \pi)$. The billiard map preserves the measure $\mu=\sin (\theta) \mathrm{d} \theta \wedge \mathrm{d} t$, see, e.g. [8].

Rychlik's proof was simplified in [5,7,9], see also a survey paper [3]. In [5], Wojtkovski proved Rychlik's theorem using Jacobi fields. He first proved that if there is a neighbourhood containing only three-period orbits, then the identity

$$
k(t) \cdot L(t, \theta)=2 \sin ^{3}(\theta)
$$


must hold, where $k(t)$ is the boundary curvature and $L(t, \theta)$ is the length of the three-period orbit $(t, \theta)$. By Fermat's principle, the length is extremized by the actual orbits, and thus $L(t, \theta)$ is constant for any continuous family of orbits. The contradiction is easily derived by observing that, by varying $\theta$, the right-hand side will change while the left-hand side will be constant. Wojtkovski, following Rychlik [6], extended the contradiction to the case when the set $P^{3}$ has positive Lebesgue measure. The argument relies on the presence of a Lebesgue density point in whose neighbourhood one can differentiate the above identity in $\theta$, arriving at a contradiction again.

A natural question arises as to how optimal this result is. Clearly, periodic orbits can form a one-parameter family, e.g., in a billiard with circular boundary there is a one-dimensional set of three-period orbits, so the Hausdorff dimension can be equal to one. But can this set have the Hausdorff dimension between one and two? Note that for the two-period orbits the answer is clearly no, since such orbits must be normal to the boundary. Thus, $\theta=\pi / 2$ and the set is confined to the one-dimensional line segment.

Our main result states that a similar sharp bound holds for the three-period orbits.

Theorem 1.1. If the billiard boundary is $\mathcal{C}^{3}$, then the Hausdorff dimension of the set of threeperiod orbits $P^{3}$ is not more than one, i.e.

$$
\mathcal{H}^{s}\left(P^{3}\right)=0 \quad \text { if } s>1,
$$

where $\mathcal{H}^{s}$ denotes the Hausdorff $s$-dimensional measure.

In the case when the Hausdorff dimension is equal to one, there is more information about the structure of the set $P^{3}$, exhibited by the following theorem. See section 3 for the definitions.

Theorem 1.2. Assume the billiard boundary is $\mathcal{C}^{3}$ and $\operatorname{dim}_{\mathcal{H}^{1}}\left(P^{3}\right)=1$. Then the set $P^{3}$ has a tangent line at $\mathcal{H}^{1}$-almost every point $p \in P^{3}$.

Note that in the case of two-period orbits the analogous statement holds for the above reason: $P^{2}$ is confined to the line segment $\theta=\pi / 2$.

The paper is organized as follows. Section 2 contains an elementary lemma on existence of a sequence converging to an accumulation point of a given set and that is asymptotic to a certain direction. It also provides an elementary formula for a directional derivative of a map via a sequence of points accumulating asymptotically in the given direction. In section 3 we define and develop the relevant notions from measure theory, mostly based on [1]. In the last section we apply those results to prove main theorems on the structure of the set of three-period orbits.

\section{Derivatives along sets}

For two points $p$ and $q$ in $\mathbb{R}^{2}$, we denote by $|p-q|$ the Euclidean distance between them. If $E$ and $F$ are two subsets of $\mathbb{R}^{2}$, we denote by $\operatorname{dist}(E, F)$ the distance between them. We also denote by $\operatorname{diam}(E)$ the Euclidean diameter of a set $E \subseteq \mathbb{R}^{2}$. Finally, let $B(p, r)$ denote the closed disc in $\mathbb{R}^{2}$ centred at $p$ of radius $r \geqslant 0$.

For $\gamma \in \mathbb{R}$ and $\rho, \eta>0$, we consider the circular sector

$$
W_{p}(\gamma ; \rho, \eta)=\left\{q=p+t \mathrm{e}^{\mathrm{i} \theta}: 0 \leqslant t \leqslant \rho,|\theta-\gamma| \leqslant \eta\right\} .
$$

Lemma 2.1. Let $E$ be a subset of the disc $B(p, r) \subseteq \mathbb{R}^{2}$. Assume that $F$ is a closed subset of the boundary circle $\partial B(p, r)$ such that $p$ is an accumulation point for $E \cap C_{F}$, where $C_{F}$ is the cone over $F$, i.e.

$$
C_{F}=\left\{q \in B(p, r): q=p+t \mathrm{e}^{\mathrm{i} \theta}, r \mathrm{e}^{\mathrm{i} \theta} \in F, t \in[0, r]\right\} .
$$


Then there exist a ray $R$, emanating from $p$ and passing through a point in $F$, and a sequence $\left(p_{k}\right)$ with $p_{k} \in E \backslash\{p\}, \lim p_{k}=p$, such that $\left(p_{k}\right)$ is asymptotic to $R$, i.e.

$$
\lim _{k \rightarrow \infty} \frac{\operatorname{dist}\left(p_{k}, R\right)}{\left|p_{k}-p\right|}=0 \text {. }
$$

Proof. For every $n \in \mathbb{N}$, we consider the open cover

$$
\mathcal{G}_{n}=\left\{U_{n}(\gamma): r \mathrm{e}^{\mathrm{i} \gamma} \in F\right\}
$$

of $F$, where

$$
U_{n}(\gamma)=\left\{r \mathrm{e}^{\mathrm{i} \theta}: \theta \in\left(\gamma-1 / 2^{n}, \gamma+1 / 2^{n}\right)\right\}
$$

Since $F$ is compact, $\mathcal{G}_{n}$ contains a finite subcover, denoted $\mathcal{G}_{n}(F)$. Let $\mathcal{G}_{n}(F)=$ $\left\{U_{n}\left(\gamma_{n}^{1}\right), \ldots, U_{n}\left(\gamma_{n}^{N(n)}\right)\right\}$.

We assumed that $p$ is an accumulation point for $E \cap C_{F}$. Therefore, for every $n \in \mathbb{N}$, there exists $\gamma_{n} \in\left\{\gamma_{n}^{1}, \ldots, \gamma_{n}^{N(n)}\right\}$, such that $p$ is an accumulation point for

$$
E \cap W_{p}\left(\gamma_{n} ; 1 / n, 1 / 2^{n}\right) \text {. }
$$

Moreover, we may assume that for each $n \in \mathbb{N}$, we have $r \mathrm{e}^{\mathrm{i} \gamma_{n+1}} \in \overline{U_{n}\left(\gamma_{n}\right)}$, the closure of $U_{n}\left(\gamma_{n}\right)$. Indeed, $F_{n}=F \cap \overline{U_{n}\left(\gamma_{n}\right)}$ is a compact set such that $p$ is an accumulation point for $E \cap C_{F_{n}}$. Thus the sequence $\left(r \mathrm{e}^{\mathrm{i} \gamma_{n}}\right)$ converges to $r \mathrm{e}^{\mathrm{i} \gamma_{\infty}}$. Note that $r \mathrm{e}^{\mathrm{i} \gamma_{\infty}} \in F$.

Let $R$ be the ray emanating from $p$ and passing through the point $r \mathrm{e}^{\mathrm{i} \gamma_{\infty}}$. From the above, we know that there exists a sequence $\left(p_{n}\right), p_{n} \in E \backslash\{p\}$, such that

$$
p_{n} \in W_{p}\left(\gamma_{n} ; 1 / n, 1 / 2^{n}\right) .
$$

Since $\lim 1 / n=0$, we conclude that $\lim p_{n}=p$. Finally,

$$
\frac{\operatorname{dist}\left(p_{n}, R\right)}{\left|p_{n}-p\right|} \leqslant \sin \left(\frac{1}{2^{n}}+\sum_{j=0}^{\infty} \frac{1}{2^{n+j}}\right)=\sin \left(\frac{3}{2^{n}}\right) \rightarrow 0, \quad k \rightarrow \infty .
$$

The following lemma is elementary.

Lemma 2.2. Let $p$ be an arbitrary point in $\mathbb{R}^{2}$ and let $U \subseteq \mathbb{R}^{2}$ be a neighbourhood of $p$. Suppose that $F: U \rightarrow \mathbb{R}$ or $\mathbb{R}^{2}$ is a $\mathcal{C}^{1}$-differentiable map in $U$. Let $\boldsymbol{v}$ be a unit vector in $\mathbb{R}^{2}$ and $R$ be a ray emanating from $p$ in the direction of $v$. We assume that $\left(p_{k}\right)$ is a sequence in $U \backslash\{p\}$, such that $\lim _{k \rightarrow \infty} p_{k}=p$ and $\left(p_{k}\right)$ is asymptotic to $R$ in the sense of lemma 2.1 . Then for the directional derivative $F_{v}(p)=D_{p} F(v)$, where $D_{p} F$ denotes the differential of $F$ at $p$, we have

$$
F_{v}(p)=\lim _{k \rightarrow \infty} \frac{F\left(p_{k}\right)-F(p)}{\left|p_{k}-p\right|} .
$$

Proof. For $k \in \mathbb{N}$, let $q_{k}$ be the projection of $p_{k}$ onto $R$. Then

On the other hand,

$$
F_{v}(p)=\lim _{k \rightarrow \infty} \frac{F\left(q_{k}\right)-F(p)}{\left|q_{k}-p\right|} .
$$

$$
\frac{F\left(p_{k}\right)-F(p)}{\left|p_{k}-p\right|}=\frac{F\left(q_{k}\right)-F(p)}{\left|q_{k}-p\right|(1+o(1))}+\frac{F\left(p_{k}\right)-F\left(q_{k}\right)}{\left|p_{k}-p\right|}, \quad k \rightarrow \infty,
$$

because $\left(p_{k}\right)$ is asymptotic to $R$. Also, since $F \in \mathcal{C}^{1}$ in $U$, it is Lipschitz in a neighbourhood of $p$. Therefore there exists $L>0$ such that

$$
\left|F\left(p_{k}\right)-F\left(q_{k}\right)\right| \leqslant L\left|p_{k}-q_{k}\right|=L \operatorname{dist}\left(p_{k}, R\right),
$$

for all $k$ large enough. By passing to the limit in (1) and using the assumption that $\left(p_{k}\right)$ is asymptotic to $R$, we obtain the desired formula for the directional derivative $F_{v}(p)$. 


\section{Measure theory}

Let $E \subseteq \mathbb{R}^{2}$ and $0 \leqslant s<\infty$. For $\delta>0$ we define

$$
\mathcal{H}_{\delta}^{s}(E)=\inf \sum_{i \in I} \operatorname{diam}^{s}\left(U_{i}\right),
$$

where the infimum is taken over all covers $\left\{U_{i}: i \in I\right\}$ of $E$ with $0<\operatorname{diam}\left(U_{i}\right) \leqslant \delta, i \in I$. The Hausdorff $s$-dimensional outer measure of $E$ is

$$
\mathcal{H}^{s}(E)=\lim _{\delta \rightarrow 0+} \mathcal{H}_{\delta}^{s}(E)
$$

The Hausdorff $s$-dimensional outer measure is indeed an outer measure, i.e. $\mathcal{H}^{s}(\emptyset)=0, \mathcal{H}^{s}$ is monotone and subadditive. It is also a metric outer measure, i.e. if $E$ and $F$ are two subsets of $\mathbb{R}^{2}$ with $\operatorname{dist}(E, F)>0$, then

$$
\mathcal{H}^{s}(E \cup F)=\mathcal{H}^{s}(E)+\mathcal{H}^{s}(F) .
$$

Therefore we have the following consequence.

Lemma 3.1 ([1, theorem 1.5]). All Borel sets are $\mathcal{H}^{s}$-measurable.

It is easy to see that for any $E \subseteq \mathbb{R}^{2}$, the function $\mathcal{H}^{s}(E)$ is non-increasing in $s$. Furthermore, there exists a unique value $s_{0}$ such that $\mathcal{H}^{s}(E)=\infty$ for $0 \leqslant s<s_{0}$ and $\mathcal{H}^{s}(E)=0$ is $s>s_{0}$. The value $s_{0}$ is called the Hausdorff dimension of $E$, and it is denoted $\operatorname{dim}_{\mathcal{H}}(E)$. An $s$-set $E \subseteq \mathbb{R}^{2}$ is an $\mathcal{H}^{s}$-measurable set with $0<\mathcal{H}^{s}(E)<\infty$. It is immediate that if $E$ is an $s$-set, its Hausdorff dimension is $s$.

Theorem 3.2 ([1, theorem 5.4(a)]). Let $E \subseteq \mathbb{R}^{2}$ be a closed set with $\mathcal{H}^{s}(E)=\infty$. Then for every $c>0$, there exists a compact subset $F \subseteq E$ such that $\mathcal{H}^{s}(F)=c$. In particular, $F$ is an s-set.

If $E$ is a $\mathcal{H}^{s}$-measurable set in $\mathbb{R}^{2}$, we say that $F \subseteq E$ is a full measure subset if $\mathcal{H}^{s}(E \backslash F)=0$. Also, if $E$ is a $\mathcal{H}^{s}$-measurable set, we say that a property holds at $\mathcal{H}^{s}$ almost all points of $E$, or at $\mathcal{H}^{s}$-almost every point of $E$, if there exists a full measure subset $F \subseteq E$ such that the property holds at every point of $F$.

If $E \subseteq \mathbb{R}^{2}$ is a $\mathcal{H}^{s}$-measurable set and $p \in \mathbb{R}^{2}$, the lower and upper density of $E$ at $p$ are defined as

$D_{s}(E, p)=\liminf _{r \rightarrow 0+} \frac{\mathcal{H}^{s}(E \cap B(p, r))}{(2 r)^{s}}, \quad D^{s}(E, p)=\limsup _{r \rightarrow 0+} \frac{\mathcal{H}^{s}(E \cap B(p, r))}{(2 r)^{s}}$,

respectively. If $E$ is an $s$-set, a point $p \in E$ such that $D_{s}(E, p)=D^{s}(E, p)=1$ is called a regular point of $E$. Otherwise $p$ is called an irregular point.

Lemma 3.3 ([1, corollary 2.5$])$. If $E$ is an s-set in $\mathbb{R}^{2}$, then

$$
\frac{1}{2^{s}} \leqslant D^{s}(E, p) \leqslant 1
$$

at $\mathcal{H}^{s}$-almost all $p$ in $E$.

For $\gamma \in \mathbb{R}$ and $\eta>0$, we also define the upper angular density of $E$ at $p$ as

$$
D^{s}(E, p, \gamma, \eta)=\limsup _{r \rightarrow 0+} \frac{\mathcal{H}^{s}\left(E \cap W_{p}(\gamma ; r, \eta)\right)}{(2 r)^{s}} .
$$

A $\mathcal{H}^{s}$-measurable set $E \subseteq \mathbb{R}^{2}$ has a tangent line at $p$ in direction $\gamma \in \mathbb{R}$ if $D^{s}(E, p)>0$ and if for every $\eta>0$,

$$
\lim _{r \rightarrow 0+} \frac{\mathcal{H}^{s}\left(E \cap\left(B(p, r) \backslash\left(W_{p}(\gamma ; r, \eta) \cup W_{p}(\gamma+\pi ; r, \eta)\right)\right)\right)}{r^{s}}=0 .
$$


We recall that $W_{p}(\gamma ; \rho, \eta)$ is the circular sector

$$
\left\{q=p+t \mathrm{e}^{\mathrm{i} \theta}: 0 \leqslant t \leqslant \rho, \gamma-\eta \leqslant \theta \leqslant \gamma+\eta\right\} .
$$

Lemma 3.4 ([1, lemma 4.5]). If $1<s<2$ and $E$ is an $s$-set in $\mathbb{R}^{2}$, then for $\mathcal{H}^{s}$-almost all $p \in E$ we have

$$
D^{s}(E, p, \gamma, \eta) \leqslant 4 \times 10^{s} \eta^{s-1}
$$

for all $\gamma \in \mathbb{R}$ and $\eta \leqslant \pi / 2$.

Corollary 3.5. If $1<s<2$ and $E$ is an $s$-set in $\mathbb{R}^{2}$, then at $\mathcal{H}^{s}$-almost all points of $E$ no tangent line exists.

Proof. The sub-additivity of $\mathcal{H}^{s}$ gives

$$
\begin{aligned}
\mathcal{H}^{s}(E \cap B(p, r)) & \leqslant \mathcal{H}^{s}\left(E \cap W_{p}(\gamma ; r, \eta)\right)+\mathcal{H}^{s}\left(E \cap W_{p}(\gamma+\pi ; r, \eta)\right) \\
& +\mathcal{H}^{s}\left(E \cap\left(B(p, r) \backslash\left(W_{p}(\gamma ; r, \eta) \cup W_{p}(\gamma+\pi ; r, \eta)\right)\right)\right)
\end{aligned}
$$

for all $\gamma \in \mathbb{R}$ and $\eta>0$.

Suppose that $E$ has a tangent line $\gamma$ at $p$. Then, by dividing both sides of (2) by $(2 r)^{s}$ and taking lim sup as $r \rightarrow 0+$, we conclude that

$$
D^{s}(E, p) \leqslant D^{s}(E, p, \gamma, \eta)+D^{s}(E, p, \gamma+\pi, \eta)
$$

for all $\gamma \in \mathbb{R}$ and $\eta>0$. By applying lemma 3.4, we further obtain

$$
D^{s}(E, p) \leqslant 8 \times 10^{s} \eta^{s-1}
$$

for all $\eta \leqslant \pi / 2$ and $\mathcal{H}^{s}$-almost all $p \in E$ such that $E$ has a tangent line at $p$. Since $s>1$, this gives $D^{s}(E, p)=0$. An application of lemma 3.3 concludes the proof.

Corollary 3.6. If $1<s<2$ and $E$ is an $s$-set in $\mathbb{R}^{2}$, then at $\mathcal{H}^{s}$-almost all points $p \in E$ we have

$$
0<\limsup _{r \rightarrow 0+} \frac{\mathcal{H}^{s}\left(E \cap\left(B(p, r) \backslash\left(W_{p}(\gamma ; r, \eta) \cup W_{p}(\gamma+\pi ; r, \eta)\right)\right)\right)}{r^{s}} \leqslant 2^{s}
$$

for all $\gamma \in \mathbb{R}$ and some $\eta>0$.

Proof. The right-hand side inequality follows from the monotonicity of $\mathcal{H}^{s}$ and lemma 3.3. The left-hand side inequality holds for every $p \in E$ such that $D^{s}(E, p)>0$ and $E$ has no tangent line at $p$. Lemma 3.3 and corollary 3.5 imply that the set of such points has full $\mathcal{H}^{s}$-measure.

Theorem 3.7. Let $E \subseteq \mathbb{R}^{2}$ be an $s$-set, $1<s<2$. Then for $\mathcal{H}^{s}$-almost every point $p$ of $E$ there exist two rays $R_{1}$ and $R_{2}$, emanating from $p$ and not contained in the same line, and two sequences $\left(p_{k}\right)$ and $\left(q_{k}\right)$ in $E \backslash\{p\}$, such that $\lim p_{k}=\lim q_{k}=p$, the sequence $\left(p_{k}\right)$ is asymptotic to $R_{1}$, and the sequence $\left(q_{k}\right)$ is asymptotic to $R_{2}$.

Proof. Let $p \in E$ be an arbitrary point satisfying the two inequalities of corollary 3.6 for all $\gamma \in \mathbb{R}$ and some $\eta>0$. That corollary states that the set of such points has full $\mathcal{H}^{s}$-measure.

Let $\gamma \in \mathbb{R}$ be arbitrary. Then there exists $\eta>0$ such that

$$
\limsup _{r \rightarrow 0+} \frac{\mathcal{H}^{s}\left(E \cap\left(B(p, r) \backslash\left(W_{p}(\gamma ; r, \eta) \cup W_{p}(\gamma+\pi ; r, \eta)\right)\right)\right)}{r^{s}}>0 .
$$


We may assume that $\eta<\pi / 2$. In particular, for a fixed $r>0$, $p$ is an accumulation point for

$$
E \cap\left(W_{p}(\gamma+\pi / 2 ; r, \pi / 2-\eta) \cup W_{p}(\gamma-\pi / 2 ; r, \pi / 2-\eta)\right) .
$$

By lemma 2.1, there exist a ray $R_{1}$, emanating from $p$ and passing through a point in

$$
F=\partial B(p, r) \cap\left(W_{p}(\gamma+\pi / 2 ; r, \pi / 2-\eta) \cup W_{p}(\gamma-\pi / 2 ; r, \pi / 2-\eta)\right),
$$

and a sequence $\left(p_{k}\right)$ with $p_{k} \in E \backslash\{p\}, \lim p_{k}=p$, such that $\left(p_{k}\right)$ is asymptotic to $R_{1}$. Clearly $R_{1}$ is not contained in the line through $p$ and $r \mathrm{e}^{\mathrm{i} \gamma}$.

Let $\gamma^{\prime} \in \mathbb{R}$ be such that $R_{1}$ contains the point $r \mathrm{e}^{\mathrm{i} \gamma^{\prime}}$. Then we can apply the same argument as above for $\gamma^{\prime}$ in place of $\gamma$ to produce a ray $R_{2}$ and a sequence $\left(q_{k}\right)$ as in the statement of the theorem. The theorem follows.

Theorem 3.8. Let $U \subseteq \mathbb{R}^{2}$ be an open set and $F: U \rightarrow \mathbb{R}^{2}$ be a $\mathcal{C}^{1}$-differentiable map. Let $E \subseteq U$ be a closed subset that consists of all fixed points of $F$. We assume that the Hausdorff dimension of $E$ is $s, 1<s \leqslant 2$. Then for every $s^{\prime}, 1<s^{\prime}<s$, there exists an $s^{\prime}$-set $E^{\prime} \subseteq E$, such that $D_{p} F=$ id for every $p \in E^{\prime}$.

Proof. Let $s^{\prime}, 1<s^{\prime}<s$ be arbitrary. Then $\mathcal{H}^{s^{\prime}}(E)=\infty$, and therefore theorem 3.2 implies that there exists an $s^{\prime}$-set $E_{1} \subseteq E$. Since $1<s^{\prime}<2$, theorem 3.7 yields the existence of a full measure set $E^{\prime} \subseteq E_{1}$ with the following property. For each point $p \in E^{\prime}$ there are two rays $R_{1}$ and $R_{2}$, emanating from $p$ and not contained in the same line, and two sequences $\left(p_{k}\right)$ and $\left(q_{k}\right)$ in $E^{\prime} \backslash\{p\}$ with $\lim p_{k}=\lim q_{k}=p,\left(p_{k}\right)$ is asymptotic to $R_{1}$, and $\left(q_{k}\right)$ is asymptotic to $R_{2}$.

Let $v_{1}$ and $v_{2}$ be the unit vectors that give the directions of $R_{1}$ and $R_{2}$, respectively. Note that $\boldsymbol{v}_{1}$ and $\boldsymbol{v}_{2}$ are linearly independent. According to lemma 2.2, for every $p \in E^{\prime}$,

$$
F_{\boldsymbol{v}_{1}}(p)=\lim _{k \rightarrow \infty} \frac{F\left(p_{k}\right)-F(p)}{\left|p_{k}-p\right|}=\lim _{k \rightarrow \infty} \frac{p_{k}-p}{\left|p_{k}-p\right|}=\boldsymbol{v}_{1},
$$

since $E^{\prime} \subseteq E$, and $\left(p_{k}\right)$ is asymptotic to $R_{1}$. Likewise, $F_{\boldsymbol{v}_{2}}(p)=\boldsymbol{v}_{2}$.

Since $\boldsymbol{v}_{1}$ and $\boldsymbol{v}_{2}$ form a basis in $\mathbb{R}^{2}$, for every $\boldsymbol{v}$ in $\mathbb{R}^{2}$ we have $\boldsymbol{v}=c_{1} \boldsymbol{v}_{1}+c_{2} \boldsymbol{v}_{2}$, where $c_{1}, c_{2} \in \mathbb{R}$. Thus, for every $p \in E^{\prime}$,

$D_{p} F(\boldsymbol{v})=c_{1} D_{p} F\left(\boldsymbol{v}_{1}\right)+c_{2} D_{p} F\left(\boldsymbol{v}_{2}\right)=c_{1} F_{\boldsymbol{v}_{1}}(p)+c_{2} F_{\boldsymbol{v}_{2}}(p)=c_{1} \boldsymbol{v}_{1}+c_{2} \boldsymbol{v}_{2}=\boldsymbol{v}$,

i.e. $D_{p} F=\mathrm{id}$.

Using a similar argument, one can prove the following result.

Theorem 3.9. Let $U \subseteq \mathbb{R}^{2}$ be an open set and $f, g: U \rightarrow \mathbb{R}$ be two $\mathcal{C}^{1}$-differentiable functions. We assume that $E \subseteq U$ is an s-set for $1<s<2$ and $f=g$ on $E$. Then there exists a full $\mathcal{H}^{s}$-measure subset $E^{\prime} \subseteq E$ with the following property. For each $p \in E^{\prime}$ there are two linearly independent unit vectors $\boldsymbol{v}_{1}$ and $\boldsymbol{v}_{2}$, such that $f_{\boldsymbol{v}_{j}}(p)=g_{\boldsymbol{v}_{j}}(p), j=1,2$.

Proof. Let $E^{\prime} \subseteq E$ be the full measure set that comes from theorem 3.7, and let $p \in E^{\prime}$ be arbitrary. Let $R_{1}$ and $R_{2}$ be two rays, emanating from $p$ and that are not contained in the same line. Let $v_{1}$ and $v_{2}$ be the linearly independent unit vectors that give the directions of $R_{1}$ and $R_{2}$, respectively. Let $R_{j}, j=1$ or 2 , be one of the rays, and $\left(p_{k}\right), p_{k} \in E^{\prime} \backslash\{p\}, \lim p_{k}=p$, be an asymptotic sequence to $R_{j}$. Then, by applying lemma 2.2 , we obtain

$$
f_{v_{j}}(p)=\lim _{k \rightarrow \infty} \frac{f\left(p_{k}\right)-f(p)}{\left|p_{k}-p\right|}=\frac{g\left(p_{k}\right)-g(p)}{\left|p_{k}-p\right|}=g_{v_{j}}(p),
$$

as desired. 


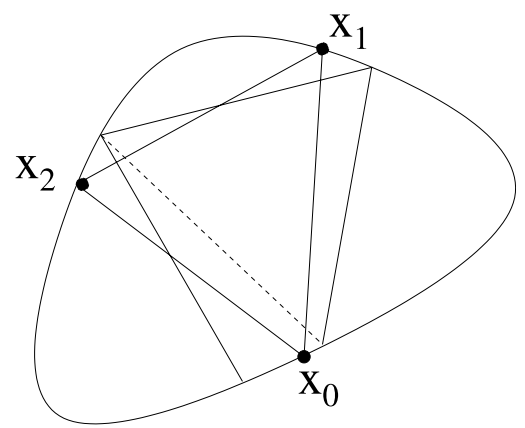

Figure 1. The periodic orbit $\left(x_{0}, x_{1}, x_{2}\right)$ has a well-defined length function $L$ (the perimeter) A nearby orbit does not have to be three-periodic. To extend the length function to such orbits, replace the third segment with the one connecting the second reflection point with the starting point (dashed line). The extended length function is then the perimeter of the obtained triangle.

We note that the main ingredient of the proofs of theorems 3.8 and 3.9 is the existence of sets of points at which no tangent line exists. Therefore we immediately have the following, more general, results.

Theorem 3.10. Let $U \subseteq \mathbb{R}^{2}$ be an open set and $F: U \rightarrow \mathbb{R}^{2}$ be a $\mathcal{C}^{1}$-differentiable map. Further, let $E \subseteq U$ be a closed subset that consists of all fixed points of $F$. Then $D_{p} F=\mathrm{id}$ at every $p \in E$ such that $E$ has no tangent line at $p$.

Theorem 3.11. Let $U \subseteq \mathbb{R}^{2}$ be an open set and $f, g: U \rightarrow \mathbb{R}$ be $\mathcal{C}^{1}$-differentiable functions. Then for every $p \in E$ such that $E$ does not have a tangent line at $p$, there are two linearly independent unit vectors $\boldsymbol{v}_{1}$ and $\boldsymbol{v}_{2}$, such that $f_{\boldsymbol{v}_{j}}(p)=g_{\boldsymbol{v}_{j}}(p), j=1,2$.

\section{Proofs of the main results}

Assume that $\mathcal{H}^{s}\left(P^{3}\right) \neq 0$ for some $s \in(1,2]$. On the set $P^{3} \subseteq U$ we have $T^{3}(p)=p$. We may assume that the annulus $U=S^{1} \times I$ is smoothly embedded in the plane. We still denote the local coordinates by $(t, \theta)$. Since the billiard boundary is $\mathcal{C}^{3}$, an elementary argument gives that the map $T^{3}$ is $\mathcal{C}^{2}$-differentiable, in particular $D_{p} T^{3}$ exists at each $p \in U$. We need the following result.

Proposition 4.1 ([5, section 4]). If $T^{3}(t, \theta)=(t, \theta)$ and $D_{(t, \theta)} T^{3}=\mathrm{id}$, then

$$
k(t) L(t, \theta)=2 \sin ^{3}(\theta),
$$

where $k(t)$ is the boundary curvature at $t$ and $L(t, \theta)$ is the length of the three-period orbit $(t, \theta)$.

The function $L$ in the above statement is only defined on $P^{3}$. However, since the billard boundary is smooth, the function $L$ can be extended to a $\mathcal{C}^{1}$-differentiable function in a neighbourhood of every three-period orbit. Indeed, one can just replace the second reflection with the straight line connecting the second collision point $\left(x_{2}\right)$ with the initial point $\left(x_{0}\right)$; see figure 1.

Now, since the billiard boundary is $\mathcal{C}^{3}$, both sides of equation (3) are defined and $\mathcal{C}^{1}$-differentiable in an open set containing $P^{3}$. Note, however, that, in general, equation (3) does not have to hold in this whole open set. 


\subsection{Proof of theorem 1.1}

Assume to the contrary that $\operatorname{dim}_{\mathcal{H}}\left(P^{3}\right)=s$ with $1<s \leqslant 2$. Note that the set $P^{3}$ is closed. Then, by theorem 3.8, for any $s^{\prime}, 1<s^{\prime}<s$, there exists a set $E^{\prime} \subseteq P^{3}$, such that $D_{(t, \theta)} T^{3}=$ id for any $(t, \theta) \in E^{\prime}$. According to proposition 4.1, on this set, the identity

$$
k(t) L(t, \theta)=2 \sin ^{3}(\theta)
$$

holds.

We apply theorem 3.9 to $E^{\prime} \subseteq P^{3}$ and the functions $f(t, \theta)=k(t) L(t, \theta), g(t, \theta)=$ $2 \sin ^{3}(\theta)$. It gives a full $\mathcal{H}^{s^{\prime}}$-measure subset $E^{\prime \prime} \subseteq E^{\prime}$ such that there are two linearly independent unit vectors $\boldsymbol{v}_{1}$ and $\boldsymbol{v}_{2}$ with $f_{\boldsymbol{v}_{j}}(p)=\bar{g}_{\boldsymbol{v}_{j}}(p), j=1,2$, for any $p \in E^{\prime \prime}$. In particular, since $f$ and $g$ are $\mathcal{C}^{1}$-differentiable, we have the equality of the partial derivatives:

$$
\frac{\partial}{\partial \theta}(k(t) L(t, \theta))=\frac{\partial}{\partial \theta}\left(2 \sin ^{3}(\theta)\right)
$$

This leads to a contradiction since $\partial_{\theta} L(t, \theta)=0$ by Fermat's principle and $\partial_{\theta} \sin ^{3}(\theta)=0$ only if $\theta=0, \pi / 2, \pi$. However, there are no three-period orbits for these values of $\theta$.

\subsection{Proof of theorem 1.2}

We may assume that $0<\mathcal{H}^{1}\left(P^{3}\right) \leqslant \infty$. Let $E \subseteq P^{3}$ be the set that consists of all points $p$ such that $P^{3}$ does not have a tangent line at $p$. If $\mathcal{H}^{1}(E)=0$, we are done. If $\mathcal{H}^{1}(E)>0$, the proof follows the lines of the proof of theorem 1.1, where one should replace theorems 3.8 and 3.9 by theorems 3.10 and 3.11 , respectively.

\section{Acknowledgment}

SM was supported by NSF grant DMS-1001144. VZ was supported by NSF grant DMS0807897.

\section{References}

[1] Falconer K J 1986 The Geometry of Fractal Sets (Cambridge Tracts in Mathematics vol 85) (Cambridge: Cambridge University Press) xiv $+162 p p$

[2] Glutsyuk A A and Kudryashov Yu G 2011 On quadrilateral orbits in planar billiards Dokl. Math. 83 371-3

[3] Gutkin E 2003 Billiard dynamics: a survey with the emphasis on open problems Regul. Chaotic Dyn. 8 1-13

[4] Ivrii V Ya 1980 The second term of the spectral asymptotics for a Laplace-Beltrami operator on manifolds with boundary Funct. Anal. Appl. 14 98-106

[5] Wojtkovski M P 1994 Two applications of Jacobi fields to the billiard ball problem J. Diff. Geom. 40 155-64

[6] Rychlik M 1989 Periodic orbits of the billiard ball map in a convex domain J. Diff. Geom. 30 191-205

[7] Stojanov L 1991 Note on the periodic points of the billiard J. Diff. Geom 34 835-7

[8] Tabachnikov S 2005 Geometry and Billiards (Student Mathematical Library vol 30) (Providence, RI: American Mathematical Society)

[9] Vorobets Y 1994 On the measure of the set of periodic points of a billiard Mat. Notes 55 455-60 